\title{
Criminologie
}

\section{Points de vue de femmes incarcérées dans des établissements fédéraux concernant les impacts du contexte carcéral sur leur trajectoire de consommation de drogues}

\section{Chantal Plourde, Serge Brochu, Amélie Couvrette et Annie Gendron}

Volume 40, numéro 1, printemps 2007

Conduites addictives et crimes

URI : https://id.erudit.org/iderudit/016017ar

DOI : https://doi.org/10.7202/016017ar

Aller au sommaire du numéro

Éditeur(s)

Les Presses de l'Université de Montréal

ISSN

0316-0041 (imprimé)

1492-1367 (numérique)

Découvrir la revue

Citer cet article

Plourde, C., Brochu, S., Couvrette, A. \& Gendron, A. (2007). Points de vue de femmes incarcérées dans des établissements fédéraux concernant les impacts du contexte carcéral sur leur trajectoire de consommation de drogues.

Criminologie, 40(1), 105-134. https://doi.org/10.7202/016017ar
Résumé de l'article

La consommation de substances psychoactives (SPA) chez les femmes détenues ne fait que très rarement l'objet de recherches. Pourtant, l'usage de SPA chez les femmes judiciarisées avant leur incarcération est fréquent. Le matériel qualitatif traité dans le cadre de cet article de même que les analyses qui en découlent visent à améliorer notre compréhension des trajectoires de consommation des femmes incarcérées et à aborder des dimensions essentielles de la vie de ces femmes qui méritent d'être mieux comprises des intervenants et des chercheurs. À partir d'une recherche à devis mixte s'intéressant aux trajectoires de consommation des femmes détenues (avant et pendant l'incarcération) dans cinq établissements carcéraux du Canada, trois thèmes reliés à l'expérience d'enfermement carcéral en rapport avec la consommation de drogues ont émergé des discours : le moment charnière, la trêve, les blâmes. L'exercice fut réalisé à partir des propos de 38 femmes ayant à la fois affirmé éprouver un quelconque problème avec l'alcool ou une autre drogue avant leur incarcération et ayant abordé spontanément la question de l'effet de l'incarcération sur leur cheminement en regard de la consommation.
Ce document est protégé par la loi sur le droit d'auteur. L'utilisation des services d'Érudit (y compris la reproduction) est assujettie à sa politique d'utilisation que vous pouvez consulter en ligne.

https://apropos.erudit.org/fr/usagers/politique-dutilisation/ 


\title{
Points de vue de femmes incarcérées dans des établissements fédéraux concernant les impacts du contexte carcéral sur leur trajectoire de consommation de drogues ${ }^{1}$
}

\author{
Chantal Plourde \\ Professeure agrégée \\ Département de psychoéducation, Université du Québec à Trois-Rivières \\ chantal.plourde@uqtr.ca
}

\section{Serge Brochu}

Vice-doyen aux ressources humaines et à la gestion

Faculté des arts et des sciences

Professeur titulaire

École de criminologie, Université de Montréal

serge.brochu@umontreal.ca

\section{Amélie Couvrette}

Candidate au doctorat

École de criminologie, Université de Montréal

amelie.couvrette@umontreal.ca

Annie Gendron

Candidate au doctorat

Département de psychologie, Université du Québec à Trois-Rivières

annie.gendron@uqtr.ca

RÉSUMÉ - La consommation de substances psychoactives (SPA) chez les femmes détenues ne fait que très rarement l'objet de recherches. Pourtant, l'usage de SPA chez les femmes judiciarisées avant leur incarcération est fréquent. Le matériel qualitatif traité dans le cadre de cet article de même que les analyses qui en

1. Nous tenons à remercier le Fonds québécois de la recherche sur la société et la culture (FQRSC) et l'Université du Québec à Trois-Rivières (Fonds institutionnel de recherche) pour leur contribution financière à ce projet de recherche. Les opinions et conclusions présentées ici ne constituent pas nécessairement celles du Service correctionnel du Canada et du Ministère du Solliciteur général du Canada. 
découlent visent à améliorer notre compréhension des trajectoires de consommation des femmes incarcérées et à aborder des dimensions essentielles de la vie de ces femmes qui méritent d'être mieux comprises des intervenants et des chercheurs. $\grave{A}$ partir d'une recherche à devis mixte s'intéressant aux trajectoires de consommation des femmes détenues (avant et pendant l'incarcération) dans cinq établissements carcéraux du Canada, trois thèmes reliés à l'expérience d'enfermement carcéral en rapport avec la consommation de drogues ont émergé des discours: le moment charnière, la trêve, les blâmes. L'exercice fut réalisé à partir des propos de 38 femmes ayant à la fois affirmé éprouver un quelconque problème avec l'alcool ou une autre drogue avant leur incarcération et ayant abordé spontanément la question de l'effet de l'incarcération sur leur cheminement en regard de la consommation.

ABSTRACT - Substance use amongst women inmates has rarely been a subject of research. Yet, psychoactive substance (PAS) use is frequent before their incarceration. Qualitative data and its subsequent analysis aim at furthering our understanding of women inmates' trajectories of alcohol and drug use that need to be better understood by practicians and researchers. From a study of mixed methodology interested in trajectories of PAS use in women inmates (before and during incarceration) from five federal incarceration establishments in Canada, three themes linked to the experience of detention in relation to PAS use emerged from the narratives; the turning point, a truce, blaming. Analysis was based on the narratives of 38 women who both stated having a problem before their incarceration with alcohol, or other drugs and who spontaneously spoke about the impact their detention had had on a personnel process as regards PAS use.

Cet article propose une réflexion sur l'impact de l'incarcération sur la trajectoire de consommation de drogues ${ }^{2}$ des femmes. La question de l'enfermement des femmes et celle des profonds changements en la matière depuis les années 1990 ont fait l'objet de plusieurs publications et analyses. On ne peut en dire autant en regard des recherches sur la consommation de substances psychoactives (SPA) chez les femmes judiciarisées. Par ailleurs, trop peu nombreux sont les travaux ayant adopté une méthodologie qualitative pour mieux comprendre leur réalité. L'étude à partir de laquelle le présent article fut rédigé concerne les changements qu'opère l'incarcération dans la trajectoire de consommation de SPA des femmes détenues. Les points de vue à partir desquels nous avons élaboré cet article s'organisent autour de trois thématiques qui ont émergé des propos des répondantes ayant spontanément mis en

2. Le terme «drogue» est utilisé dans ce texte comme synonyme de substances psychoactives. Il fait donc référence à l'alcool, au tabac, aux drogues illégales et aux médicaments psychotropes prescrits ou non. 
relief leur trajectoire de consommation et leur arrivée en milieu carcéral. Ce contenu traduit des expériences influencées par différents processus subjectifs, dont le cadre institutionnel dans lequel elles sont prises en charge. Nous tenterons avec cet article d'apporter des éléments d'analyse témoignant de la complexité des interactions en cause.

\section{Problématique}

Statistiques et tendances démographiques observées dans les milieux carcéraux

Le profil démographique des individus en prison au Canada, en Europe et aux États-Unis témoigne d'une progression de la population carcérale féminine (Bertrand, 2002; Pate, 2002; Mullen et al., 2003; Staton et al., 2003). Aux États-Unis, le nombre de femmes détenues a augmenté de $336 \%$ depuis 1980, tandis que le nombre d'hommes a augmenté de $189 \%$ (Baletka et Shearer, 2001). Au Canada, une augmentation du nombre de femmes incarcérées est également observée. Le pourcentage de femmes admises en pénitencier ${ }^{3}$ a doublé comparativement à ce qu'il était il y a vingt-cinq ans, passant ainsi de $2 \%$ en 1976 à $5 \%$ en 2001 (Bertrand, 2003). Plusieurs éléments peuvent possiblement contribuer à expliquer l'augmentation du nombre de femmes incarcérées, notamment une probable évolution des comportements délictueux des femmes, la désinstitutionalisation des hôpitaux psychiatriques, l'évolution des pratiques judiciaires et l'augmentation du nombre de places consécutive à la récente réforme du système carcéral pour les femmes (Faith, 2002; Pate, 2002; Bertrand, 2003).

\section{Caractéristiques des femmes en prison}

Les femmes américaines incarcérées sont généralement des femmes économiquement défavorisées, sans emploi, âgées en moyenne entre 30 et 35 ans, d'origine afro-états-unienne dans plus de la moitié des cas, et mères d'enfants de moins de 18 ans pour les deux tiers d'entre elles (Mullings et al., 2001 ; Loper, 2002; Phillips et al., 2002; Mullen et al., 2003 ; Surratt, 2003). Les caractéristiques de femmes détenues sous responsabilité fédérale canadienne diffèrent de la population carcérale

3. Le système fédéral gère les sentences de deux ans et plus alors que les systèmes provinciaux et territoriaux sont responsables des sentences de moins de deux ans. 
états-unienne. En 1999, la plupart des femmes détenues sous responsabilité fédérale étaient âgées entre 20 et 34 ans (56\%). Elles étaient majoritairement de race blanche $(57,7 \%), 21,1 \%$ d'entre elles étaient autochtones et la plupart avaient connu un ou plusieurs événements de victimisation avant leur incarcération. Les crimes violents représentaient les principaux délits pour lesquels elles purgeaient une sentence $(65 \%)$ (Bertrand, 2002; Blanchard, 2002; Cyrenne, 2002; Frigon, 2002). Les deux tiers des femmes étaient mères et la plupart d'entre elles se trouvaient dans une situation de monoparentalité (54\% selon Hairston, $1990 ; 57 \%$ selon Withers, 2002) et représentaient le seul soutien de leur(s) enfant(s) (Eljdupovic-Guzina, 1999).

\section{Données sur la consommation de drogues des personnes incarcérées} (bommes et femmes)

Il est connu que l'utilisation de drogues est beaucoup plus élevée chez les personnes judiciarisées que dans la population en général, de même que la polytoxicomanie et la consommation de médicaments (Brochu et al., 1996; Walters, 1996; Baletka et Shearer, 2001; Langner et al., 2002; Boys et al., 2002). Les données recueillies seront présentées et mises en relief, tout d'abord à partir des travaux généraux portant sur la consommation des populations incarcérées (hommes et femmes) avant leur réclusion, puis pendant celle-ci.

Une recherche australienne menée dans 27 établissements de détention indique que $64 \%$ des répondants ont rapporté avoir déjà consommé des drogues illicites au cours de leur vie $(62 \%$ d'hommes et $71 \%$ de femmes). Plus de femmes que d'hommes rapportent avoir déjà consommé ou avoir consommé régulièrement de l'héroïne, de la cocaïne, et de la méthadone dans les 12 mois précédant l'incarcération (Butler et al., 2003). Une autre étude australienne montre que $61 \%$ des femmes $(\mathrm{n}=$ 56) avaient un problème de consommation diagnostiqué au moment de leur arrestation (Denton, 1994). Aux État-Unis, lorsque interrogés sur leur consommation antérieure à la détention, plus de $75 \%$ des détenus ont affirmé avoir consommé une drogue illicite au cours de leur vie (Harlow, 1991). Par ailleurs, Plourde (2000) rapporte que $91 \%$ des répondants incarcérés dans les pénitenciers québécois ont déjà consommé une drogue illicite au cours de leur vie et que près de $70 \%$ d'entre eux ont consommé dans les trois mois précédant leur réclusion.

En ce qui concerne spécifiquement les femmes judiciarisées, une étude réalisée par Brochu et ses collaborateurs (1992) révèle qu'elles 
consommeraient davantage que leurs homologues masculins, et que le type de substance privilégié serait distinct des produits de choix des hommes. D'après les résultats d'une étude épidémiologique sur la consommation de substances psychoactives des femmes détenues au Québec (Brochu et al., 1996), 94,7\% des répondantes ont fait l'essai d'au moins une drogue, incluant l'alcool, au cours de leur vie. L'alcool est le produit le plus consommé, suivi de la cocaïne et du cannabis. En ce qui a trait aux taux de dépendance à l'alcool et aux drogues, 14\% des femmes auraient un taux de dépendance modéré, élevé ou grave à l'alcool alors que près de $59 \%$ auraient un taux de dépendance modéré, élevé ou grave aux autres drogues.

Plusieurs auteurs ont démontré une certaine constance des patrons de consommation lors de l'incarcération (Keene, 1997; Plourde, 2000), celle-ci n'étant pas un gage d'abstinence. En effet, des recherches attestent que beaucoup de prisonniers continuent de consommer une fois incarcérés (Plourde et Brochu, 2002a et 2002b; Boys et al., 2002; Butler et al., 2003; Andía et al., 2005). Une étude menée dans les pénitenciers fédéraux québécois démontre que $33 \%$ des détenus auraient consommé une substance psychoactive dans les trois mois d'incarcération ayant immédiatement précédé l'étude (Plourde et Brochu, 2002a et 2002b). Par ailleurs, dans une étude visant à identifier les facteurs contribuant à la consommation de drogues dans une prison de New York et une de Puerto Rico, parmi les 796 participants, $41 \%$ (New York) et $38 \%$ (Puerto Rico) ont reconnu avoir consommé de la drogue lors de leur séjour en détention (Andía et al., 2005). Entre le tiers et la moitié des individus qui consommaient des drogues injectables avant leur détention continueraient de le faire en prison (Plourde et Brochu, 2002a et 2002b; Butler et al., 2003). Au Canada, une étude révèle que près de $11 \%$ des détenus ont admis avoir consommé par injection depuis qu'ils sont incarcérés (CCLAT, 2004).

Facteurs de risque associés à la consommation de SPA en prison (bommes et femmes)

À l'instar de Plourde et Brochu (2002a et 2002b), certaines études, dont celles de Boys et al. (2002) et de Gillepsie (2005), montrent que l'utilisation de drogues avant l'incarcération de même que la dépendance sont des facteurs fortement reliés à la consommation entre les murs. D'autres éléments, tels le nombre de séjours en prison et les problèmes de santé mentale du détenu, apparaissent comme des déterminants importants 
de l'usage de drogues en prison (Boys et al., 2002). Des auteurs brésiliens ont identifié comme facteurs de prédiction de la consommation entre les murs les carences quant aux opportunités d'éducation et d'emploi, une insertion précoce dans le marché de la drogue ainsi qu'une dynamique familiale déficiente (Lazaro de Carvalho et al., 2005). L'affiliation à un gang (avant la détention) et la durée de l'incarcération seraient également associées à la consommation entre les murs (Andía et al., 2005). D'autre part, les analyses de Butler et al. (2003) démontrent que les facteurs associés à un risque élevé de recourir à l'injection en prison sont le fait d'être de sexe féminin, d'être âgé de 24 à 40 ans, d'avoir été placé en foyer d'accueil pendant l'enfance, d'avoir séjourné en prison à plusieurs reprises et d'être incarcéré pour un crime violent (Butler et al., 2003).

\section{Caractéristiques des femmes toxicomanes en prison}

Des chercheurs américains (Langan et Pelissier, 2001) se sont intéressés aux besoins spécifiques des femmes et des hommes toxicomanes incarcérés. Leurs résultats montrent que la situation des femmes, quant à leur consommation, est plus détériorée que celle des hommes. Elles consomment plus souvent de manière quotidienne, elles sont plus souvent engagées dans un usage quotidien de deux drogues et plus, et dans un usage journalier d'héroïne et de cocaïne. En ce qui concerne la motivation à consommer, les femmes disent consommer pour soulager une douleur physique ou psychologique tandis que les hommes donnent des raisons d'ordre hédoniste (plaisir), ce qui rejoint les résultats de chercheurs canadiens (Plourde et Brochu, 2002a et 2002b). Ces différences témoignent possiblement de besoins distincts quant à l'offre de traitement en regard de la toxicomanie.

Denton (1994) trace un portrait plutôt sombre des caractéristiques des femmes qui consomment en prison: jeunes, célibataires, sans emploi, mères, ayant une santé précaire et une histoire d'abus physique et sexuel pour une grande proportion. En ce qui concerne leur cheminement en regard de la consommation, la précocité est une caractéristique prédominante, alors que plusieurs consomment des drogues en prison, parfois par intraveineuse (Denton, 1994). Enfin, d'après Mullings et al. (2001), les femmes incarcérées qui s'injectent constituent une population distincte qui aurait des caractéristiques et des besoins particuliers. Les résultats de l'étude soutiennent l'hypothèse d'un modèle séquentiel où la violence 
pendant l'enfance mènerait à une immersion dans le monde de la drogue, puis à un manque d'opportunités relié à l'occupation de rôles conventionnels et enfin à l'adoption d'un mode de vie chaotique, où elles commettent des actes criminels et s'initient à l'injection de drogues.

\section{Contexte carcéral et adaptation}

Il est connu qu'en dépit des améliorations dans les conditions de détention, la personne incarcérée fait face à une série de privations et de souffrances auxquelles elle doit tenter de s'adapter du mieux possible (Plourde, 2000). Les effets de l'incarcération peuvent être abordés selon plusieurs angles, notamment en regard des impacts directs vécus par la personne durant sa réclusion (santé physique et mentale, stress posttraumatique, etc.) puis en regard des dommages collatéraux consécutifs à l'incarcération et lors du retour en collectivité (Liebling et Maruna, 2005). La santé mentale et physique des individus incarcérés est menacée par les difficultés associées à un séjour en détention et les risques accrus de transmission de maladies (Tonry et Pertesilia 1999; Lalande, 2006). Les détenus seraient plus vulnérables en début d'incarcération, ceci les rendant davantage susceptibles de développer des problèmes émotionnels et des symptômes de dépression (Zamble et Porporino, 1988). Alors que ces auteurs soutiennent l'idée selon laquelle l'adaptation du détenu se ferait progressivement et que sa détresse initiale diminuerait au fur et à mesure que le temps passe, d'autres auteurs affirment que l'adaptation des détenus se caractérise par le retrait et l'évitement des contacts sociaux ou des activités dans la prison (Vacheret et Lemire, 1998).

Peu d'études se sont attardées précisément à l'adaptation des femmes lors de leur séjour en détention (Tonry et Petersilia, 1999). Il est cependant connu que les conséquences de la séparation d'avec leur famille se traduisent fréquemment par de la détresse, de la colère, de l'anxiété, de la tristesse, de la honte, de la culpabilité et un fort sentiment de perte consécutif à la séparation (Stanley et Byrne, 2000; Blanchard, 2002; Lafortune et al., 2004). La séparation et la perte des droits parentaux pourraient troubler certaines détenues, au point d'affecter alors leurs capacités d'adaptation à leur nouvelle situation et, du même coup, leurs chances de réhabilitation (Blanchard, 2002). Liebling (1999) ajoute même que la perte des droits parentaux serait un facteur associé au risque de suicide chez la femme détenue. 


\section{Cadre théorique}

Notre cadre théorique est composé de deux influences principales qui balisent depuis leur commencement nos travaux sur la consommation de drogues des hommes et des femmes incarcérés. Tout d'abord, les acteurs dans le champ de la psychologie et des services sociaux savent pertinemment que l'individu devant affronter des changements dans son homéostasie (tel un arrêt de la consommation de drogues ou l'emprisonnement) est susceptible de vivre un état de stress et qu'il devra y «faire face» en s'ajustant le mieux possible à la situation vécue (Lazarus et Folkman, 1984; Seyle, 1953; Dumont et Plancherel, 2001). Le modèle transactionnel sur le stress psychologique de Lazarus (1966), en s'intéressant à l'influence bidirectionnelle entre les ressources d'une personne et les contraintes inhérentes au milieu, permet de mieux comprendre les effets du stress sur le bien-être d'un individu.

Les stratégies adaptatives utilisées par l'individu devant faire face à une situation stressante peuvent lui permettre, dans la mesure où elles sont efficaces, de s'adapter, de diminuer l'inconfort psychologique, de conserver une image positive de soi, et de maintenir une relation satisfaisante avec son milieu de vie (Dumont, 2001). Le concept de stratégie adaptative (coping) se trouve au cour du modèle transactionnel de Lazarus et Folkman (1984), entre l'évaluation d'une situation jugée stressante et l'outcome (détresse ou adaptation). Ces stratégies font appel aux compétences individuelles utilisées pour faire face à la source de stress, qu'elle soit positive ou négative. Ainsi, le coping se résumerait aux efforts cognitifs, comportementaux et émotionnels déployés par un individu pour moduler l'effet de la source de stress sur le bien-être personnel (physique et psychologique). Puisqu'ils font partie d'un processus dynamique d'influence réciproque, ces derniers peuvent se modifier avec le temps et selon le contexte, favorisant une meilleure adaptation (Dumont, 2001; Dumont et al., 2004).

Lazarus et Folkman (1984) proposent un modèle qui repose sur deux styles de coping: l'un centré sur le problème et l'autre centré sur les émotions. Alors que le premier style de coping aspire à une modification de la source du problème par des efforts pour le résoudre (en développant de nouvelles habiletés, en le réévaluant positivement, en cherchant des informations et du soutien social), le second s'apparenterait davantage à de l'évitement en impliquant des efforts (cognitifs, comportementaux ou émotionnels), tels le déni, la pensée magique, la distraction mentale ou 
encore la consommation de substances psychoactives, déployés par l'individu pour diminuer sa détresse psychologique. Cette forme de coping (centré sur les émotions-évitements), malgré le fait qu'elle ne procure pas de sentiment de maîtrise, permet néanmoins d'envisager différemment la situation ou de la dédramatiser afin de trouver un réconfort psychologique et une forme d'optimisme plutôt que d'envisager le pire (Dumont, 2001). Si certaines de ces stratégies peuvent s'avérer utiles temporairement, lorsque la personne n'est pas encore prête à faire face à ses problèmes, il faut néanmoins savoir que l'évitement peut, à moyen terme, aggraver une situation et faire augmenter le niveau d'anxiété. Dumont (2001) rappelle que l'efficacité du coping varierait selon les circonstances et un style actif serait davantage efficace dans le cas où la personne estime avoir du contrôle alors que les stratégies d'évitement (centré sur les émotions), en permettant un répit psychologique, seraient plus bénéfiques dans des contextes où les éléments sont hors de contrôle pour l'individu (tel le contexte carcéral). Dans l'esprit de la théorie transactionnelle, l'utilisation d'une stratégie adaptative plutôt qu'une autre dépendrait entre autres du sentiment d'auto-efficacité personnel, de caractéristiques de la personnalité comme l'optimisme et le tempérament, des ressources familiales et environnementales, et du sens accordé aux événements (Dumont, 2001 ; Dumont et al., 2004).

L'ensemble de notre démarche de recherche repose également sur une deuxième source d'influence, soit celle des trajectoires de consommation. Nous faisons ici référence aux situations objectives et subjectives qui délimitent le cheminement ayant mené et maintenu l'individu dans une consommation problématique, telle qu'elle est définie par le point de vue subjectif de la personne elle-même, tout en référant aux forces qui sont en interaction dynamique et qui impriment un mouvement au devenir des individus (Bertaux, 1997; Dubar, 1998). Il apparait essentiel d'introduire les notions d'interactions entre les personnes, de contexte et de durée dans la compréhension des cheminements puisque la consommation s'inscrit dans le temps et parce que son évolution est hétéroclite (Brochu et al., 2002; Brunelle et Cousineau, 2005 ; Bertrand et Nadeau, soumis). La notion de trajectoire fait ainsi référence à une dimension dynamique qui permet de situer la personne à un point de rencontre, qui implique des déterminants externes et internes qui se modifient au fil du temps. Elle réfère en quelque sorte aux états décrits par un système dans le temps, lesquels s'enchaînent en fonction de décisions et de la subjectivité de l'acteur. 
La position des chercheurs à l'égard de l'objet étudié dans le cadre de cette recherche se situe dans la tradition subjectiviste ou interprétative (Giordano, 2003: 20) selon laquelle l'individu est un «sujet interprétant et ses interprétations lui sont spécifiques, car intimement liées à son expérience personnelle du monde». En somme, cette recherche s'intéresse à la compréhension des femmes face à leur consommation, au sens qu'elles accordent aux situations objectives et subjectives dans leur parcours de consommation.

\section{Méthodologie}

Afin de répondre aux objectifs de cette recherche à devis mixte, des entretiens ont été réalisés dans cinq pénitenciers fédéraux canadiens pour femmes (Établissement Joliette [QC], Grand Valley Institution [ON], Edmonton Institution for Women [ED], Nova Institution [NE], Fraser Valley Institution [BC]). Sur une base volontaire, 176 femmes ont été rencontrées et interrogées, à partir d'un instrument maison adapté, sur leur trajectoire de consommation d'alcool et d'autres drogues (Plourde, 2000). Cet outil emprunte des questions à des instruments validés tels l'Alcohol Dependence Scale (ADS) (Horn et al., 1984), le Questionnaire informatisé sur le mode de vie (QIMV) (Weekes et al., 1982), le Questionnaire de Santé Québec (Institut de la statistique, 1990-1991), le Mandatory Drug Testing in Prisons (MDT) (Edgar et O'Donnel, 1998), de même que l'échelle psychologique intégrale de l'Indice de gravité d'une toxicomanie (IGT) (Bergeron et al., 1992). Un article traitant spécifiquement des changements qu'induit l'incarcération sur la consommation des femmes (matériel quantitatif) est actuellement en préparation (Plourde et al.).

Nous avons donc tenté de mieux comprendre la réalité et le point de vue des femmes en leur posant la question suivante en seconde partie d'entretien: «J'aimerais que vous me parliez de manière détaillée de la signification qu'a pris pour vous la consommation de médicaments, de drogues et d'alcool jusqu'à présent». Une grille constituée de mots clés fut utilisée par les intervieweuses afin d'alimenter les entretiens qui ont pris la forme d'entrevues semi structurées (Mayer et Ouellet, 1991). Après avoir retranscrit tous les entretiens enregistrés numériquement, codifié le matériel à partir des thèmes ayant émergé (codification par émergence), réalisé quatre accords interjuges, nous avons amorcé l'ana- 
lyse du contenu des entretiens (L'Écuyer, 1990). L'analyse thématique (Ghiglione et Matalon, 1978) nous a permis d'amorcer la réduction du matériel de manière verticale (analyse de chacun des entretiens) puis de manière transversale (permettant de cibler les points de convergence et de divergence entre les entretiens). En parcourant l'ensemble du matériel, nous avons remarqué que plusieurs femmes faisaient spontanément référence à leur expérience subjective de l'incarcération et à son impact sur leur trajectoire de consommation. À travers les analyses effectuées, nous avons également relevé des points de vue diversifiés, qui portent sur différents autres impacts de l'incarcération. Nous avons donc choisi de présenter ce matériel qui aborde des dimensions essentielles de la vie de ces femmes (lesquelles méritent d'être mieux comprises des intervenants et des chercheurs) dans une logique qui respecte à la fois nos intérêts de recherche en lien avec la consommation de substances psychoactives et le discours de ces femmes sur l'essentiel, soit leur logique de compréhension face à leur vécu.

\section{Portrait des femmes dont le propos est retenu dans le cadre de cet article}

De façon plus spécifique, nous avons retenu les propos de 38 femmes sur les 176 de l'échantillon total ayant abordé spontanément au cours de l'entretien la question des impacts de l'incarcération sur leur cheminement en regard de leur consommation de substances psychoactives. Le tableau suivant fait état des caractéristiques de ces 38 femmes incarcérées. Les répondantes rapportant avoir déjà eu une consommation

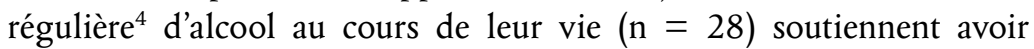
commencé à consommer de façon régulière à partir de 16 ans en moyenne. En ce qui concerne les femmes ayant rapporté avoir consommé régulièrement d'autres drogues au cours de leur vie $(\mathrm{n}=36)$, l'âge moyen du début de la consommation régulière s'avère également de 16 ans. Selon leur point de vue, $79 \%(n=30)$ avaient un problème grave $^{5}$ de consommation d'alcool ou d'autres drogues, trois mois avant leur incarcération.

4. Consommation régulière: avoir consommé au moins une fois par semaine pendant au moins un mois.

5. Les femmes devaient situer leur perception de la gravité de leur problème de consommation sur un continuum (échelle Likert) allant de «aucun problème» à «grave problème». 
TABLEAU 1

\section{Caractéristiques des participantes $(n=38)$}

Âge $M=35,89$ ans (é. t. $=8,01$ )

Origine ethnique

Canadienne: $68,4 \%(n=26) \quad$ Autochtone: $21,1 \%(n=8) \quad$ Autres: $10,4 \%(n=4)$

Statut civil

Célibataire: $57,9 \%(n=22) \quad$ Mariée: $2,6 \%(n=1) \quad$ Conjointe de fait: $15,8 \%(n=6)$

Séparée/Divorcée: $18,4 \%(n=7)$ Veuve: $5,3 \%(n=2)$

Enfant

Aucun: $15,8 \%(n=6) \quad$ Un: $31,6 \%(n=12) \quad$ Deux: $28,9 \%(n=11)$

Trois: $10,5 \%(n=4) \quad$ Quatre ou plus: $13,2 \%(n=5)$

Sentence $M=62,42$ mois (é. t. $=66,36$ )

Nombre de mois écoulés entre le début de l'incarcération et l'entretien

$M=27,66$ mois (é. t. $=36,30$ )

Nombre de mois écoulés depuis l'entrée dans le pénitencier actuel et l'entretien

$M=16,74$ mois (é. t. $=25,10$ )

Nombre de séjours en institution fédérale

$M=1,32$ fois (é. t. $=0,66$ )

Délit principal pour lequel la répondante était incarcérée

Crime contre la personne: $68 \%(n=26)$

Infraction contre les biens: $16 \%(n=6)$

Infraction en matière de stupéfiants: $11 \%(n=4)$

Autres: $5 \%(n=2)$

Âge de la consommation régulière

Alcool $=16$ ans (é. t. $=4,27$ )

$(\mathrm{n}=28)$

Autres drogues $=16,22$ ans (é. t. $=6,51$ )

$(n=36)$

Perception d'un problème de consommation d'alcool ou de drogues trois mois avant l'incarcération

Grave: $78,9 \%(n=30)$

Moyen: $7,9 \%(n=3)$

Petit: $10,5 \%(n=4)$

Non disponible $(\mathrm{n}=1)$

Tel qu'en fait foi le tableau 2, la majorité des répondantes étaient incarcérées à l'Établissement Joliette (29\%), à l'Edmonton Institution for Women (24\%) ou à la Fraser Valley Institution (21\%). L'anglais était la langue parlée par la plupart des femmes de notre échantillon $(68 \%)$. 
TA B LEA U 2

Provenance et langue des participantes

\begin{tabular}{lcccccc}
\hline Pangue & $\begin{array}{c}\text { Établissement } \\
\text { Joliette }\end{array}$ & $\begin{array}{c}\text { Grand Valley } \\
\text { Institution }\end{array}$ & $\begin{array}{c}\text { Edmonton } \\
\text { Institution } \\
\text { for Women }\end{array}$ & $\begin{array}{c}\text { Nova } \\
\text { Institution }\end{array}$ & $\begin{array}{c}\text { Fraser Valley } \\
\text { Institution }\end{array}$ & Total \\
\hline Français & $11(28,9 \%)$ & 0 & 0 & $1(2,6 \%)$ & 0 & $12(31,5 \%)$ \\
\hline Anglais & 0 & $5(13,2 \%)$ & $9(23,7 \%)$ & $4(10,5 \%)$ & $8(21,1 \%)$ & $26(68,2 \%)$ \\
\hline Total & $11(28,9 \%)$ & $5(13,2 \%)$ & $9(23,7 \%)$ & $5(13,1 \%)$ & $8(21,1 \%)$ & $38(100 \%)$ \\
\hline
\end{tabular}

\section{Les impacts de l'incarcération sur la trajectoire de consommation des femmes incarcérées}

Choisir de présenter les perspectives des participantes selon des frontières que nous avons choisies de nommer à partir des termes utilisés par celles-ci ne signifie en rien que les répondantes avaient un point de vue délimité et catégorisé quant aux impacts de leur incarcération sur leur trajectoire, au contraire. Leurs points de vue et perceptions s'avéraient pour le moins diversifiés et parfois même entremêlés, illustrant la complexité d'expliquer à autrui leur compréhension de la situation. Nous pouvons seulement constater que l'exercice auquel nous nous sommes prêtés à partir de l'importante somme de matériel qualitatif que nous avons analysé nous ramène essentiellement à ces trois réservoirs de représentations, réservoirs parfois communiquant, il faut le reconnaitre. Ainsi, le travail d'analyse nous amène à développer trois thèmes reliés à l'expérience d'enfermement carcéral en rapport avec la consommation de drogues: le moment charnière, la trêve, les blâmes.

Notre démarche maintenant exposée, il faut bien comprendre également que le propos des femmes s'inscrit dans une réalité bien particulière, soit celle du cadre institutionnel dans lequel elles sont prises en charge. Il fut précisé plus tôt que notre conceptualisation du mot «trajectoire» prend en compte les notions d'interactions interpersonnelles, de contexte et de durée dans la compréhension des dépendances parce que celles-ci s'inscrivent à la fois dans le temps et l'espace, et parce que leur évolution n'est pas nécessairement régulière. Les propos des femmes rencontrées, lesquels s'avèrent des reconstructions de leur histoire, prennent leur sens dans le cadre carcéral et il est fort possible qu'ils auraient été bien différents à l'extérieur de celui-ci. Certaines formules ou discours sont ainsi possiblement influencés par le contact entretenu avec des professionnels du milieu. En ce sens, la prison semble parfois 
se situer en «événement marquant» qui ouvre sur un processus de changement. Il en sera davantage question dans la discussion.

\section{Moment charnière}

Décrire ce premier niveau d'expérience de la rencontre de la trajectoire de consommation avec la réclusion comme un «point tournant» ou un «moment charnière» consiste à rendre compte du fait que plusieurs femmes ont un point de vue éloquent sur l'impact de cet événement dans leur vie. Selon ce qui émerge des discours, il semble que leur niveau de consommation était rendu à un point tel que leur vie était en danger certes, mais aussi leur intégrité physique et psychologique, relativement au style de vie mené et à la prise de risques importants.

Estelle $\left(21\right.$ ans, $1^{\text {re }}$ sentence fédérale, ayant purgé 10 mois sur sa sentence de 24 mois, code de sécurité: médium) est mère d'une fille qui était placée par les services sociaux au moment de l'entretien et d'un garçon dont sa mère s'occupe. Elle est accusée de vols et de voies de fait. Pour elle, le fait d'aller en prison l'a possiblement empêchée de mourir.

Si je n'étais venue en prison comme je l'ai fait, je serais probablement morte [...] Je crois que Dieu m'a sauvé la vie et je crois que le système m'a sauvé la vie aussi. De me faire arrêter de prendre de la drogue et des choses, je n'étais plus en contrôle. Et j'avais besoin d'arrêter tu sais. Donc, en m'arrêtant, ça m'a fait arrêter de consommer, ça m’a donné ce temps. J'ai été sobre depuis que j'ai été arrêtée.

Même chose pour Solange (44 ans, $4^{\mathrm{e}}$ sentence fédérale, ayant purgé 19 mois sur sa sentence de 48 mois, code de sécurité: médium) qui explique que, sans cette sentence qu'elle purge actuellement à la suite d'une mise en commun des accusations qui pesaient contre elle, «je me serais probablement suicidée, parce que j'étais tellement partie loin». Elle ajoute également qu'à l'époque de son incarcération, en plus de son style de vie marqué par des prises de risque et une détresse importante, son niveau de consommation était rendu à un tel point que si cela n'avait cessé, elle en serait morte. Pour elle, le fait de se retrouver obligée de rompre avec la consommation fut un événement positif puisque: «Je ne pouvais pas aller en désintoxication, la désintox n'aurait pas aidé, parce que je pouvais partir. Alors voilà, la prison était parfaite.»

Pour sa part, Suzan (42 ans, $2^{\mathrm{e}}$ sentence fédérale, ayant purgé neuf mois sur sa sentence de 27 mois, code de sécurité: médium) évoque l'hypothèse que le pénitencier pourrait possiblement l'avoir sauvée. 
Je vivais juste pour ça dans le fond, me geler là. Je ne voulais pas rien voir de la vie, la seule affaire qui, ce qui m'a sauvée, c'est peut-être con là, mais c'est le pénitencier.

Pour Marianne (32 ans, $1^{\text {re }}$ sentence fédérale, ayant purgé un mois sur sa sentence de 226 mois, code de sécurité: médium), qui est issue d'une famille où il y avait à la fois «beaucoup de boisson et de chicanes», pour reprendre son expression, la consommation d'alcool et d'autres drogues lui permettait de s'évader d'une grande colère causée par de multiples incidents d'abus et la violence subie, mais n'était pas sans lui causer nombre de problèmes avant son incarcération. Ici, la question de la survie dépasse la réalité objective, mais relève davantage selon son propos d'une occasion de faire un choix ou de réfléchir quant à sa destinée (vivre ou mourir).

Mais maintenant, je suis en train de réaliser ça et c'est ma seule porte de sortie, je veux dire lorsque tu consommes de la drogue et de l'alcool, tu es totalement dans le trouble tout le temps. Tu peux boire, tu peux aller boire excessivement des bières, ensuite je veux dire tu es assis en prison. Comme pour moi lorsque je... lorsque j'ai sorti de prison le 13 mars (date fictive), deux jours après j'ai commis ce crime, je ne me rappelle pas de rien. Je ne sais pas ce que je faisais en prison jusqu'à ce qu' ils me disent en Cour que j'avais, j'ai su que j'avais attaqué quelqu'un et je me suis dit que ce n'était pas moi, je n'ai jamais... je ne fais pas des choses comme ça. Lorsque les témoins sont venus et ont dit que j'avais fait ça, j'ai admis que je l'avais fait. Maintenant je le fais et je pense, je remercie Dieu qui ma mise ici parce qu'Il m'a donné une chance de penser à si je voulais mourir ou vivre.

Cette position face à ce qu'a provoqué l'entrée en pénitencier sur leur trajectoire de consommation, dans une perspective qui va au-delà des drogues, mais qui se rend à une expérience ayant permis la survie (physique et psychologique), est fréquemment observée dans les discours. L'expression «m'a sauvé la vie», «si je n'étais pas venue ici je serais morte» ou «m'a peut-être sauvé la vie» (introduisant une certaine nuance sur le réel pouvoir de l'incarcération), revient à plusieurs reprises. Ce discours sur l'incarcération comme moment charnière, notamment sur la trajectoire de consommation, a des résonances qui nous apparaissent étroitement liées à des mécanismes cognitifs, lesquels permettent aux femmes de faire face à leur situation d'incarcération et du même coup à leur nouvelle situation en regard de la consommation de drogues. En référence au modèle de Lazarus et Folkman (1984), il semble plausible que le fait de faire l'effort cognitif d'accorder un sens aussi positif à 
l'incarcération puisse contribuer à l'adaptation, et que cet effort cognitif qui s'inscrit dans un processus dynamique est influencé largement par le contexte carcéral et le temps écoulé depuis le début de la sentence. De prime abord, le fait d'accorder un sens à ce point positif à l'incarcération pourrait faire référence au coping centré sur l'émotion, lequel s'apparente davantage à de l'évitement (pensée magique sur le rôle d'une puissance supérieure dans la situation) et s'avère efficace pour diminuer la détresse psychologique et dédramatiser dans un contexte où la femme a peu de pouvoir sur sa situation (Dumont, 2001). Nous remarquons également que moins de deux ans se sont écoulés (et parfois même seulement un mois) depuis le début de leur réclusion, ce qui peut influencer l'adoption de stratégies centrées sur les émotions, lesquelles pourraient à moyen terme faire en sorte d'aggraver la situation psychologique de la femme, notamment en augmentant son niveau d'anxiété (Dumont, 2001). Par ailleurs, il est également concevable que le fait, pour une femme, d'envisager la situation plus positivement (réévaluer de manière positive le fait d'être en prison) peut également s'avérer efficace comme stratégie de résolution de problème (coping centré sur le problème), mais implique que la personne peut avoir le sentiment d'avoir du contrôle sur sa situation. Évidemment, dans la mesure où elles sont efficaces pour la personne, ces différentes stratégies de coping peuvent permettre à la femme devant vivre le stress relié à une incarcération de s'adapter, de diminuer son inconfort psychologique (entre autres celui relié au changement dans son patron de consommation), de conserver une image positive d'elle-même et de maintenir une relation satisfaisante avec son milieu de vie (Dumont, 2001).

\section{La trêve}

Le deuxième type d'expérience est celui de la trêve, qu'il aurait été également possible de qualifier de «cure de désintoxication» des comportements destructeurs au sens large, et non seulement des habitudes reliées à la consommation de drogues. Les femmes nous ont largement parlé du fait que la prison (ou encore les épisodes d'incarcération pour certaines) avait été l'occasion d'un arrêt plus ou moins long, d'un espace de transition, d'un temps pour soi, iront jusqu'à dire certaines d'entre elles.

Pour Nicole (39 ans, $2^{\text {e }}$ sentence fédérale, ayant purgé 15 mois sur sa sentence de 42 mois, code de sécurité: médium), sa trajectoire de 
consommation a débuté alors qu'elle était très jeune et les périodes d'incarcération ont toujours imposé des trêves dans sa trajectoire d'usage de drogues. Des trêves que l'on pourrait qualifier de «petites cures» sporadiques, comme des périodes de jeûnes temporaires qui visent à «purger» le système.

Je suis une dépendante depuis que je suis jeune, depuis que j'ai comme 11 ans, je suis dépendante des drogues et de l'alcool. Je suis devenue une consommatrice d'opiacés lorsque j'avais 17, 18, 19 autour de ça, j'ai commencé à prendre de l'héroïne. Je me suis tenue, été dans les rues pendant 20 ans. J'ai fait beaucoup d'alcool et de drogues, j'entre et je sors du système depuis à peu près 20 ans, oui à peu près. Depuis que j'ai 16 ans que j'entre et je sors du système, alors je suis devenue... Comme je me gelais, faisais des délits, me gelais, faisais des délits, me gelais, faisais des délits, me ramasser en prison, faire des délits ou me geler, faire des délits, me ramasser en prison, sortir, me geler, faire des crimes. J'ai vécu comme ça pendant à peu près 20 ans... Finalement, j'ai eu une sentence fédérale et maintenant je suis ici et j'ai, j'ai été sobre pour un an alors...venir en prison a toujours fait diminuer mes habitudes de consommation. À chaque fois que je suis venue en prison, $j$ 'ai fait le sevrage des opiacés et hum, en accumulant de l'argent, du moment que je sortais, j'allais m'acheter de la drogue, j'allais me geler. Alors la seule fois où je n'ai jamais, que je ne faisais pas des drogues c'est lorsque que je venais en prison. Parce que c'est comme une période calme pour moi, je reste, je dors, reçois de l'aide et ressors.

Chantraine (2004: 14) a développé un système d'idéaux types des rapports à l'incarcération dans lequel se retrouve l'idéal type «incarcération break», lequel fait notamment référence à la situation où l'enfermement se pose comme «un moyen brutal certes, mais efficace, de marquer une pause régulatrice des pratiques toxicomaniaques», où l'on se refait une santé. Brassard (2004: 181) remarque également que pour les femmes autochtones plus institutionnalisées de son échantillon, la période d'incarcération sert à refaire une santé et «panser les maux causés par un style de vie instable et qui contribue à détériorer leur santé».

Alors qu'elle raconte avoir agi comme «dealer de drogues» pour financer sa propre consommation (ce qui lui a valu une incarcération de deux ans), Karen (29 ans, $1^{\text {re }}$ sentence fédérale, ayant purgé trois mois sur sa sentence de 24 mois, code de sécurité: médium) tient un propos qui laisse entendre une certaine ambivalence quant à l'impact de l'incarcération sur elle. "Je ne sais pas si c'était une si mauvaise chose, parce que c'est la première fois que je suis sobre en 10 ans et je 
regarde ça comme une cure de désintoxication maintenant.» Elle admet toutefois que la drogue lui permettait de faire face à ses problèmes, et que certaines journées elle ressent le manque et qu'elle n'est pas convaincue qu'elle pourra toujours résister.

Je ne vais pas masseoir ici et mentir que je ne suis pas en manque de temps en temps, car quand j'ai une mauvaise journée, je suis comme «Oh mon Dieu! Si j'en avais en ce moment je pense que j'en ferais». Bien je croyais sincèrement que je le ferais jusqu'à temps que récemment, il n'y a pas si longtemps, on m'a offert quelque chose et, en fait, je m'en suis éloignée. Et je ne pouvais pas le croire. C'était la première fois que je pouvais faire ça en dix ans. [...] C'était une chose difficile et je pense que si ça m'était arrivé 20 minutes plus tard, je l'aurais probablement fait, je ne le sais pas si j'aurais été capable de dire non tout de suite encore. Bien je l'ai fait et je me suis éloignée de là [...] Alors espérons que je ferai ça 100000 fois de plus peut-être et je pourrai mourir libérée des drogues, je n'en suis pas certaine.

Pour Vivian (36 ans, $1^{\text {re }}$ sentence fédérale, ayant purgé 92 mois sur sa sentence de 122 mois, code de sécurité: médium), ce n'est pas uniquement l'incarcération comme telle qui a joué un grand rôle dans sa trajectoire de consommation, mais la durée de celle-ci et les programmes dans lesquels elle s'est impliquée.

J'ai fait tous les programmes ici puis j'ai tout le temps eu des programmes réussis. Puis je n'étais pas, je n'allais pas prôner des affaires que je n'étais pas là. [...] Je prenais ce qui était bon pour moi, puis ce que je pensais qui n'était pas bon, je le laissais de côté c'est tout là. [...] J'ai joué des games tu sais, je l'ai fait aussi ça, mais à un moment donné, j'ai décroché de ça, «c'est tu ça que je veux dans ma vie?» Puis le temps fait aussi. Quand tu as une grande sentence... Parce que ce n'est pas vrai qu'une fille qui entre ici avec un problème de consommation, qu'elle ressort dans six mois et que tout est réglé [...] Si j’avais juste fait un an là, je ne serais pas en train de te dire ça aujourd'hui. Je ne te parlerais même pas comme ça, je parlerais comme une écartée, une fuckée, mais le temps a fait que ça n'a pas pris une semaine et, la première année, j'ai arrêté de consommer. Je me battais continuellement avec moi pour ne pas consommer [...] Moi ça m'a beaucoup aidée puis je suis bien, comme je te dis, je suis réellement contente d'avoir passé ici. Je suis réellement contente que ça soit fini. Puis en même temps, je suis triste de m'en aller, puis en même temps, bien là, ça me claque en pleine face, j'ai vraiment tué quelqu'un, je le sais aujourd'hui.

Jeanne (44 ans, $1^{\text {re }}$ sentence fédérale, ayant purgé plus de 70 mois de sa sentence de 72 mois, code de sécurité: médium) va dans le même 
sens quant à l'impact de la durée de la sentence sur sa réhabilitation par rapport à son problème de consommation.

J'étais vraiment triste de la sentence. J'ai eu sept ans, mais je vois maintenant pourquoi j'ai eu ça, c'était voulu que j'aie ça. Parce que si ça était plus court, je n'aurais pas été capable d'arrêter la drogue. Parce que je n'étais, je n'ai pas... Je voulais arrêter l'héroïne parce que j'étais tellement malade, ça me tuait, mais la coke, je voulais continuer, il n'y avait pas de... Tu sais, il n'y avait pas de raisons pour lesquelles je voulais arrêter, jusqu'à ce que j'arrive en prison et que je suive des programmes et que je me sente mieux. Après j'ai vu comment ça ruinait ma vie.

Ces propos traduisent l'idée que ces femmes ont utilisé les difficultés reliées à l'incarcération comme «moteur» de changement les ayant amenées à faire quelque chose de positif, à s'engager sur une voie pavée d'efforts visant la résolution de problèmes. En référence au modèle transactionnel de Lazarus et Folkman (1984), cette forme de coping centré sur le problème fait référence aux efforts orientés vers l'environnement ou envers soi visant la résolution du problème de consommation. Nous avons vu qu'il est difficile d'identifier une stratégie de coping toujours efficace dans une situation donnée puisque l'efficacité d'une stratégie dépend d'une série de facteurs, dont les ressources personnelles et environnementales de la personne (Dumont, 2001). Il semble que pour les femmes ayant manifesté une quelconque forme de mobilisation, des indices d'efficacité reliés notamment à la résolution plus ou moins permanente du problème ou encore à des états émotionnels positifs émergent des discours.

Plusieurs participantes ont un discours qui glisse vers l'idée que le simple fait de se retrouver au pénitencier ait contribué à leur processus de maturation. Ainsi, les femmes ne donnent habituellement pas le crédit aux programmes offerts dans l'institution à proprement parler, mais plutôt à l'arrêt imposé par la réclusion, faisant également référence à une évolution naturelle qui se situe au-delà de l'incarcération, ellemême parfois influencée par d'autres événements marquants.

Je ne crois pas que tu peux arrêter jusqu'à ce que tu veuilles arrêter, alors je suis allée à travers ça, tu sais, à travers plusieurs centres de traitement, plusieurs, rien n'a fonctionné. Tu n'arrêtes pas jusqu'à ce que tu le veuilles ou que tu en aies assez. (Question: Alors, qu'est-ce qui s'est passé pour que vous arrêtiez?) Hum, j'étais juste tannée de ça, et tu sais, réaliser finalement ce qui m'a menée ici et juste maturer, je regarde ça aujourd'hui et je regarde en arrière et je pense à, tu sais, mes victimes, que j'ai volé des banques, et 
tu sais, il y avait quelques-unes des femmes qui, évidemment, avaient très peur de me voir dans une banque avec un fusil, et elles ont capoté un peu. Hum, je ne sais pas, c'est juste, je ne sais pas, les choses changent, comme, j'ai juste maturé, et hum, j'ai eu l'hépatite $\mathrm{C}$ tu sais, j'étais très inquiète à propos de ça et les deux, mon mari et moi l'avons eu, et tu sais, j'ai été très malade, je suis un traitement depuis les dernières deux années. (Valérie, 38 ans, $1^{\text {re }}$ sentence fédérale, ayant purgé 31 mois de sa sentence de 96 mois, code de sécurité: médium).

Emily (23 ans, $1^{\text {re }}$ sentence fédérale, ayant purgé 18 mois de sa sentence de 30 mois, code de sécurité: médium) a cessé de consommer des drogues lorsqu'elle «est rentrée en dedans», mais elle ne veut pas donner tout le crédit à l'incarcération seulement.

À un moment donné, les valeurs changent et puis tout ça. Puis on comprend des choses puis comme je n'ai pas le goût de refaire la même chose, je n'ai pas le goût de retourner dans l'escorte ou de retourner danser ou tout ça. Je me suis dit, bien regarde, il faudrait peut-être que j'arrête à un moment donné là. Fait que c'est ça.

Que cela soit une cure sporadique, un arrêt de consommation durable, un processus de maturation personnel, cette trêve suscite pour plusieurs une réflexion certaine sur leur mode de vie et sur leur consommation. Pour d'autres, l'idée de la trêve évoque davantage un arrêt des comportements destructeurs, et il apparaît difficile d'isoler la consommation de drogues des autres conditions de vie de ces femmes. Pour elles, même si l'incarcération pèse lourd, plusieurs le mentionnent, elles essaient de tirer profit des programmes ou, du moins, du temps de réclusion forcé. Faisant référence à nouveau au modèle de Lazarus et Folkman (1984), nous remarquons dans les propos de ces femmes des formes variées de mobilisation individuelle et d'implication dans les programmes qui s'apparentent à des stratégies de coping centrées sur le problème. En ce sens, il semblerait que ce niveau de discours se retrouve davantage (mais pas exclusivement) chez des femmes ayant purgé plusieurs mois de leur sentence.

\section{Les blâmes}

Tel qu'il a été discuté en première partie de cet article, la documentation abonde quant aux nombreux impacts négatifs et à l'exacerbation des dommages que provoque l'expérience d'incarcération chez la personne 
détenue de même que sur sa famille (Liebling et Maruna, 2005). Ainsi, en plus des deux autres thématiques émergentes (moment charnière et trêve) du discours des répondantes sur cette rencontre entre la trajectoire de consommation et l'incarcération, les femmes que nous avons eu le privilège de rencontrer ont des choses à dire également à propos d'impacts moins directement liés à la consommation, mais plutôt à leur vie en général. À ces impacts est intimement liée l'idée d'un blâme dirigé à la fois envers leurs propres comportements et envers le système carcéral et social. Cette brisure de la logique interne de cet article qui porte sur la trajectoire de consommation de ces femmes nous sera pardonnée (nous l'espérons) du fait que les effets généraux de l'incarcération ont tout de même leur place puisqu'ils témoignent des traces que laisse l'expérience carcérale sur le vécu subjectif. Ces traces auront possiblement des effets sur la condition mentale et physique des femmes au moment de leur sortie et conséquemment sur leur trajectoire de consommation.

Si certaines femmes accordent du crédit à l'incarcération ou du moins à l'arrêt d'agir qui leur a été imposé par l'arrestation et ses suites, elles sont plusieurs également à avoir un point de vue marqué par l'ambivalence à ce sujet. Ainsi, tout en reconnaissant que la prison les a «sauvées» d'un gouffre quelconque, elles reconnaissent d'autres conséquences à cette expérience comme en témoigne l'extrait suivant d'une entrevue auprès de Patricia ( 35 ans, $1^{\text {re }}$ sentence fédérale, ayant purgé 26 mois de sa sentence de 28 mois, code de sécurité: minimum), qui se qualifie elle-même de cocaïnomane.

Je suis ici en prison, donc j'ai ruiné ma propre vie. Tu sais, pour le reste de ma vie, tu sais, ça va me suivre partout. Mais ça m’a aussi aidée d'une certaine façon, à arrêter les drogues, venir en prison parce que j'ai failli mourir. J'ai été, j'ai frappé un mur à $180 \mathrm{~km}$ à l'heure. J'avais $3000 \mathrm{mg}$ de dope dans mon système quand j'ai été arrêtée. Donc si ça n'était pas de tout ça et de moi venant ici, bien je n'aurais pas réalisé ce que, ce qui se passait tu sais.

Une autre femme, Isabelle (30 ans, $2^{\mathrm{e}}$ sentence fédérale, ayant purgé huit mois de sa sentence de 62 mois, code de sécurité: médium) a le point de vue suivant:

Je repense à tout ça, c'est comme... C'est fou. Je me dis: «Criss, j'aurais pu faire un meurtre là.» J'étais rendue vraiment loin dans ma tête là. C'est plus ça qui vient m'atteindre, puis que je me dis: «Tabarnak, tu as 30 ans Isabelle, c'est le temps que tu t'allumes là et que tu fasses quelque chose de 
ta criss de vie là, tu passeras pas ta vie en prison là. » Moi j'ai deux beaux enfants moi là dehors puis j'ai perdu, tu sais, la maternelle de mon gars, la première année, la deuxième année, j'ai tout perdu ça, moi, je pourrai jamais reprendre ça. Tu sais, des affaires que j'ai perdues, ma fille, je l'ai vue à la maternelle puis je l'ai vue à la première année puis le reste, j'ai tout perdu. C'est comme tu sais, j'ai perdu des beaux moments, puis ce n'est pas explicable.

À partir des entretiens que nous avons analysés, il appert que la situation délicate que crée l'incarcération sur la famille et sur la mère provoque l'émergence de sentiments douloureux associés à des pertes, de même que la certitude de «manquer quelque chose», ce qui est fidèle à ce que nous avons relevé dans les écrits (Liebling, 1999; Blanchard, 2002). Maria (30 ans) témoigne que le fait d'être séparée de ses enfants et du reste de sa famille s'avère très difficile, de même que le fait d'être consciente de tout ce qu'elle manque: «seulement recevoir des photos de mes enfants qui grandissent, les occasions que je rate». Ceci n'est pas sans la faire souffrir et lui causer des envies de consommer, d'autant plus que, compte tenu de la nature des accusations portées contre elle, elle devra «aller vivre à un autre endroit afin que ma famille ne soit pas dérangée ou harcelée».

Il y a des jours où c'est difficile lorsque je deviens vraiment frustrée lorsque je veux boire, je traverse des périodes ou j'haïs tout le monde et je souhaite que quelqu'un ait de la broue ou quelque chose à boire juste pour me saouler et tout oublier. Si je ne m'en soucie pas, mettez-moi dans un trou ou peu importe, je veux seulement prendre une brosse avec quelqu'un. Certains jours sont comme ça. Mais c'est... j'écoute ma musique ou je me tiens occupée pour ne pas être en manque. (Maria, 30 ans, $2^{\mathrm{e}}$ sentence fédérale, ayant purgé cinq mois de sa sentence de 48 mois, code de sécurité: médium).

Tel qu'il a été évoqué précédemment, les traces ou le marquage de l'incarcération sur l'individu sont préoccupants pour sa réinsertion sociale certes, mais également compte tenu des effets potentiels sur sa santé physique et psychologique. En ce sens, mais en dépassant ce premier niveau de réflexion, des questionnements nous apparaissent importants en regard du fait que cet événement marquant impose une certaine forme d'abstinence ou encore façonne la consommation de ces femmes entre les murs (Plourde et al., en préparation). Comme le souligne Jacques (1999), on ne peut s'intéresser à l'abstinence sans en aborder le processus antérieur. Ainsi, on peut se demander si le fait de 
constater une certaine forme d'abstinence des femmes incarcérées sans égard à sa signification et à sa portée sur différents plans poserait des risques interprétatifs. On peut penser que cette trêve de consommation, durant laquelle la souffrance continue de grandir, ne s'établit pas comme base problématique encore plus sérieuse pour la sortie du pénitencier. L'extrait précédent laisse soupçonner non pas un rétablissement, mais un mal qui apparait plutôt exacerbé.

Si on ne sent pas de blâme à proprement parler contre le système carcéral dans ces extraits et le matériel qui les précède, mais plutôt un blâme essentiellement dirigé contre soi-même par rapport au fait de se retrouver en prison (mais surtout, par ailleurs, une profonde déception face à ce qui a été perdu), le prochain extrait témoigne, pour sa part, d'une certaine forme de blâme qui s'adresse non pas au système carcéral en tant qu'institution, mais au pouvoir discrétionnaire (l'interviewée parle de méchanceté) d'un agent de libération qui, après avoir constaté la consommation de cette femme, fait en sorte qu'elle se retrouve encore une fois derrière les barreaux.

Mais si tu as un agent méchant comme j'ai eu [...] Quand il m’a remontée et après j'ai eu un pisse-test sale et il m'a jetée ici pour six mois. Tout ce qu'il a fait, j'ai perdu mon appartement maintenant, hum, mon emploi, tu vois ce que je veux dire? Il m'a fait reculer encore plus loin. Au lieu de dire: «Ok, bien elle a consommé, qu'est-ce que tu vas faire pour ça?», tu sais, il n'avait pas à me renvoyer directement au pénitencier... Je crois que les agents de libération conditionnelle ont besoin de... Je crois que les agents de libération conditionnelle ne devraient pas essayer d'être des intervenants en toxicomanie parce qu'ils ne sont pas des ex-toxicomanes eux-mêmes et ils ne sont pas des psychologues... Les gens qui sont des agents de libération conditionnelle devraient être, ne devraient pas simplement être des gens ordinaires, je ne crois pas. Qui ne comprennent pas ce que les femmes, les souliers dans lesquels elles marchent... Ils ne savent pas parce qu'ils retournent dans leur maison de banlieue, dans leur famille tu sais, leur salaire annuel de $40000-50000 \$$. Ils n'ont jamais eu, ils n'ont jamais marché dans nos souliers, ils n'ont jamais eu d'endroit où vivre [sic], ou qu'ils ne savent pas d'où le prochain chèque de paie arrivera et tu es trop malade pour travailler! Tu vois ce que je veux dire. Ils ne comprennent pas, ils posent trop de jugements. (Louise, 42 ans, $1^{\text {re }}$ sentence fédérale, ayant purgé 22 mois de sa sentence de 24 mois, code de sécurité: minimum)

Un autre extrait appuie cette idée de blâme du service carcéral, mais ici Jocelyne insiste sur la spécificité de la réalité féminine et sur le sens que prend l'incarcération pour une femme. 
Parce que la plupart des filles ici, à $80 \%$, elles ont été violées puis ont été abusées. Donc, quand une femme fait cinq ans là, c'est bien assez, elle a compris puis surtout quand elle a des enfants [...] Une femme, ce n'est pas comme les hommes, ils ont des sentences bonbons. Une femme là qui a des enfants dehors, qu'elle a fait un meurtre ou whatever, après cinq ans, quand elle a subi l'incarcération, elle a été brimée de tous ses droits... ses libertés et qu'elle est plus capable de voir ses enfants, celle-là est dépourvue, elle a plus le goût de vivre là. Fait que après cinq ans, [...] une femme, elle devrait retourner, avoir des maisons pour elles. Pas à l'intérieur, ça ne donne rien! (Jocelyne, 48 ans, $1^{\text {re }}$ sentence fédérale, ayant purgé 85 mois de sa sentence de 108 mois, code de sécurité: médium)

Dans le prochain extrait, on sent cette fois que le blâme est dirigé vers le système social ou l'idéologie populaire à l'égard des femmes qui commettent un délit.

Oui, la prison a changé ma vie. Je ne suis pas en train de dire que la prison est bonne, ce n'est pas ça. Les femmes ne vont pas dans les cages. Personne ne le mérite. Mais la façon que le système, hum... le public le regarde, il croit que nous devons totalement hum... être punies lorsque nous sommes ici. Ils ne savent pas comment ça nous punit juste d'être loin... juste de ne pas pouvoir marcher sur la rue ou de voir ta famille ou des choses du genre, pas vrai? (Jeanne, 44 ans, $1^{\text {re }}$ sentence fédérale, ayant purgé plus de 70 mois de sa sentence de 72 mois, code de sécurité: médium)

Il nous était impossible de passer outre à ces propos puisqu'ils ont émergé du discours de plusieurs femmes. Ainsi, si certaines se blâment pour leur incarcération ainsi que pour les séparations et les pertes qui en découlent, d'autres condamnent le système correctionnel. Enfin, pour d'autres, l'opinion populaire vis-à-vis de la femme criminelle est un des aspects dommageables de leur incarcération.

\section{Discussion et conclusions}

Notre objectif premier en rédigeant cet article était d'aborder des dimensions qui nous apparaissent essentielles de la vie des femmes incarcérées et qui, à notre avis, méritent d'être mieux comprises des intervenants et des chercheurs. Nous constatons que la question de la consommation de substances psychoactives est malheureusement très peu explorée dans la documentation traitant de l'incarcération des femmes. Cet article permet de saisir que différents changements surviennent dans la vie des femmes incarcérées en regard de la consommation 
et que l'incarcération en tant qu'événement stresseur oblige des évaluations cognitives qui permettent à l'individu de s'ajuster le mieux possible à cet événement marquant et à ce qu'il impose sur la vie et sur la trajectoire de consommation. Les stratégies adaptatives déployées par les répondantes s'inscrivent sur un continuum et font partie d'un processus dynamique marqué notamment par l'influence du temps, du contexte, de caractéristiques personnelles et du sens accordé aux événements par les acteurs. Les discours témoignent de formes variées de stratégies faisant intervenir des styles actifs (coping centré sur le problème) ou plutôt orientés vers des stratégies d'évitement (coping centré sur les émotions) en fonction de la perception face au contrôle de la situation mais qui, dans un cas comme dans l'autre, visent l'adaptation et certainement la diminution d'inconforts psychologiques. Des distinctions portant spécifiquement sur les différentes substances psychoactives (licites et illicites) viendront ajouter à la compréhension et alimenter nos analyses sur les trajectoires objectives et subjectives de consommation des femmes incarcérées, notamment la place et le rôle possible de la médication dans l'institution (Plourde et al., en préparation).

À l'instar de ce que Brassard (2004) a constaté dans le discours des femmes autochtones ayant fait l'expérience de l'enfermement carcéral, nous pourrions soutenir l'idée que les femmes que nous avons eu le privilège d'écouter ont effectué une reconstruction mentale de leurs représentations leur permettant de mieux vivre l'expérience de l'enfermement de même qu'un dépassement des conceptions essentiellement négatives de l'institution (Brassard, 2004). La perspective voulant que le séjour en prison se pose en trêve fut souvent évoquée par les femmes rencontrées. Une trêve pour leur consommation, une trêve ayant permis une survie physique et psychologique. Pour d'autres, la prison aura possiblement contribué à modifier temporairement la trajectoire de consommation, mais elle aura également concouru à briser ce qui était déjà cassé, avec les conséquences que l'on peut anticiper pour la sortie. Frigon (2006: 70) soulève l'idée que des questions sont trop souvent oubliées: «Est-ce que la prison, peut, doit, devrait guérir? Pourquoi ne pas soigner ces femmes hors des murs?»

On peut également se demander s'il est normal de constater que l'incarcération constitue en quelque sorte un refuge «salutaire» face à une consommation. Notre société prend-elle alors ses responsabilités? Peut-on croire à une pause ou à un arrêt définitif de la consommation alors que le milieu carcéral ne place pas la personne en contact avec ses 
motivations profondes à consommer? Ceci dit, en se prêtant à un exercice de dépassement du niveau d'analyse subjectif réalisé à partir du contenu brut livré par ces femmes, il devient intéressant, quoique interprétatif, de se questionner sur le réel impact de la réhabilitation en milieu carcéral, notamment dans un contexte de sortie de l'institution. Ainsi, dans un cadre de prise en charge, le discours s'avère nécessairement coloré par la réalité et celle-ci étant complètement différente de la réalité extérieure à l'institution, il est prudent de garder à l'esprit cette subjectivité qui prend son sens entre les murs tout en demeurant fidèle au point de vue de ces femmes qui nous ont livré leur témoignage, lequel pose parfois l'institution comme «salutaire». Par ailleurs, le fait que la prison en tant que telle se pose positivement en «événement marquant» pour les répondantes n'a rien d'étonnant en soi puisque de nombreux intervenants y œuvrant font tout en leur possible pour aider les personnes incarcérées en investissant toute leur énergie et leur savoir, ce qui permet certainement à des femmes de s'ouvrir sur un processus de changement.

Plusieurs études interpellent les services correctionnels face à l'importance d'un suivi thérapeutique lors de la libération, et non seulement un accompagnement qui vise la sécurité publique et la prévention de la récidive à l'ère de la justice actuarielle et un appui que l'on peut certainement qualifier de variable dans le processus de réinsertion sociale. Cette étude ne fait pas exception et ses auteurs estiment que, dans une société où la prison s'impose malheureusement trop souvent comme solution de rechange pour des individus marqués par des événements difficiles, il faut dans une perspective du «moindre mal» s'assurer que les personnes qui transigent bien malgré elles dans ces institutions soient traitées et soutenues si elles le souhaitent, et ce, du mieux possible et non seulement durant la réclusion, mais également lors de la remise en liberté.

\section{Références}

Andía, J. F., Deren, S., Robles, R. R., Kang, S. Y., Colón, H. M., Oliver-Velez, D., \& Finlinson, A. (2005). Factors associated with injection and noninjection drug use during incarceration among Puerto Rican drug injectors in New York and Puerto Rico. The prison journal, 85 (3), 329-342.

Baletka, D. M., \& Shearer, R. A (2001). Counselling female offenders who abuse substances. TCA Journal, 29 (2), 140-148. 
Bergeron, J., Landry, M., Ishak, I., Vaugeois, P., \& Trépanier, M. (1992). Validation d'un instrument d'évaluation de la gravité des problèmes reliés à la consommation de drogues et d'alcool, l'indice de gravité d'une toxicomanie (IGT). Montréal: Cahiers de recherche du RISQ.

Bertaux, D. (1997). Les récits de vie: perspectives ethnosociologiques. Paris: Nathan.

Bertrand, M.-A. (2002). Progrès, recul et stagnation. Tableau contrasté des conditions de vie des femmes incarcérées au Canada. Criminologie, 35 (2), 135-146.

Bertrand, M.-A. (2003). Les femmes et la criminalité. Montréal: Athéna Éditions.

Bertrand, K., \& Nadeau, L. (soumis). Toxicomanie et inadaptation sociale grave: perspectives subjectives de femmes en traitement quant à l'initiation et la progression de leur consommation. Drogues, Santé et Société.

Blanchard, B. (2002). La situation des mères incarcérées et de leurs enfants au Québec. Criminologie, 35, (2), 91-112.

Boys, A., Farrell, M., Bebbington, P., Brugha, T., Coid, J., Jenkins, R., Marsden, J., Meltzer, H., Singleton, N., \& Taylor, C. (2002). Drug use and initiation in prison: results from a national prison survey in England and Wales. Addiction, 97 (12), 1551-1560.

Brassard, R. (2004). L'expérience et les effets de l'enfermement carcéral des femmes autochtones au Québec. Montréal: Thèse de doctorat. Montréal, Université de Montréal.

Brochu, S., Desjardins, L., Douyon, A., \& Forget, C. (1992). Drug Use Prevalence among offenders. In F. Losel, D. Bender \& T. Bliesner (ed.), Psychology and law: International Perspectives (105-110). Berlin: Walter de Gruyter.

Brochu, S., Biron, L., \& Desjardins, L. (1996). Consommation de substances psychoactives chez les jeunes femmes détenues au Québec. Criminologie, 29 (1), 121-139.

Brochu, S., Da Agra, C., \& Cousineau, M.-M. (2002). Drugs and Crime Deviant Pathways. Burlington: Ashgate Publishing.

Brunelle, N., \& Cousineau, M.-M. (2005). Trajectoires de déviance juvénile. Les éclairages de la recherche qualitative. Sainte-Foy: Les Presses de l'Université du Québec.

Butler, T., Levy, M., Dolan, K., \& Kaldor, J. (2003). Drug use and its correlates in an Australian prisoner population. Addiction Research $\mathfrak{F}$ Theory, 11 (2), 89-101.

Centre canadien de Lutte contre l'alcoolisme et la toxicomanie (2004). FAQ sur la toxicomanie dans les établissements de correction. Ottawa.

Chantraine, G. (2004). Par-delà les murs. Expériences et trajectoires en maison d'arrêt. Paris: Presses Universitaires de France.

Cyrenne, M.-A. (2002). Réflexions sur la réalité actuelle des détenues dans le système correctionnel fédéral. Criminologie, 35 (2), 31-52.

Denton, B. (1994). Prisons, Drugs and Women: a report on the drug use of women in Victorian prisons. National Drug Strategy. Research Report Series, 5. Australie: La Trobe University. 
Dubar, C. (1998). Trajectoires sociales et formes identitaires: clarifications conceptuelles et méthodologiques. Sociétés contemporaines, 29, 73-85.

Dumont, M., \& Plancherel, B. (2001). Stress et adaptation chez l'enfant. Sainte-Foy: Les Presses de l'Université du Québec.

Dumont, M. (2001). Les stratégies adaptatives. In M. Dumont \& B. Plancherel (ed.), Stress et adaptation chez l'enfant (53-68). Sainte-Foy: Les Presses de l'Université du Québec.

Dumont, M., Pronovost, J., \& Leclerc, D. (2004). Les stratégies adaptatives des adolescents: comparaison d'un groupe scolaire et d'un groupe desservi en Centres jeunesse. Revue de Psychoéducation, 33 (1), 137-155.

Edgar, K., \& O’Donnel, I. (1998). Mandatory Drug Testing in Prison: The Relationship between MDT and the Level and Nature of Drug Misuse. London: Home Office Research Study 189, Home Office Research and Statistics Directorate.

Eljdupovic-Guzina, G. (1999). Le rôle parental et les expériences de violence des délinquantes; examen des évaluations initiales des délinquantes. Ottawa: Service Correctionnel du Canada.

Faith, K. (2002). La résistance à la pénalité: un impératif féministe. Criminologie, 35 (2), 113-134.

Frigon, S. (2006). Écorchées. Montréal: Éditions du remue-ménage.

Frigon, S. (2002). La création de choix pour les femmes incarcérées: sur les traces d'un groupe d'étude sur les femmes purgeant une peine fédérale et ses conséquences. Criminologie, 35 (2), 9-30.

Gillepsie, W. (2005). A multilevel model of drug abuse inside prison. The prison Journal, 85 (2), 223-246.

Giordano, Y. (2003). Conduire un projet de recherche: une perspective qualitative. Paris: Éditions Management \& Société.

Ghiglione, R., \& Matalon, B. (1978). Les enquêtes sociologiques, théories et pratiques. Paris: Armand Colin.

Hairston, C. F. (1990). Mothers in jail: parent-child separation and jail visitation. Affilia, 6 (2), 9-27.

Harlow, C. W. (1991). Drugs and jail inmates, 1989. Washington, DC: U.S. Department of Justice.

Horn, J. L., Skinner, H. A., Wanberg, K., \& Foster F. M. (1984) Alcobol Dependence scale. Toronto: Addiction Research Foundation.

Institut de la Statistique (1990-1991). Questionnaire Santé Québec. Québec: ministère de la Santé et des Services sociaux.

Jacques, J.-P. (1999). Pour en finir avec les toxicomanies: Psychanalyse et pourvoyance légalisée des drogues. Bruxelles: De Boeck.

Keene, J. (1997). Drug use among prisoners before, during and after custody. Addiction research, 4 (4), 343-353.

Lafortune, D., Barette, M., Dubeau, D., Bellemare, D., Brunelle, N., Plourde, C., \& Cusson, J.-F. (2004). Un père incarcéré: facteur de risque ou de protection pour ses enfants? Psychiatrie $\mathfrak{\sigma}$ Violence, 4 (2). Disponible sur le site: http://www.psychiatrieviolence.ca/archive.htm. 
Lalande P. (2006). La sévérité pénale à l’heure du populisme. Québec: Direction des communications, ministère de la Sécurité publique.

Langan, N. P., \& Pelissier, B. M. M. (2001). Gender differences among prisoners in drug treatment. Journal of Substance Abuse, 13, 291-301.

Langner, N., Barton, J., McDonnagh, D., Noël, C., \& Bouchard, F. (2002) Taux de médicaments prescrits chez les détenues. Forum, 14 (2), 10-14.

Lazaro de Carvalho, M., Gonçalves Valente, J., Gonçalves de Assis, S., \& Godoi Vasconceslos, A. G. (2005). Predictive model for cocaine use in prison in Rio de Janeiro, Brazil. Saúde Pública, 39 (5), 824-831.

Lazarus, R. S. (1966). Psychological stress and the coping process. New-York: McGraw-Hill.

Lazarus, R. S., \& Folkman, S. (1984). Stress, Appraisal, and Coping. New York: Springer.

L'Écuyer, R. (1990). Méthodologie de l'analyse développementale de contenu: méthode GPS et concept de soi. Sainte-Foy: Les Presses de l'Université du Québec.

Liebling, A. (1999). Prison, Suicide and Prisoner Coping. In M. Tonry \& J. Petersilia (ed.), Crime and Justice: A Review of Research, 26, 283-359.

Liebling, A., \& Maruna, S. (2005). The Effects of Imprisonment. Cullompton: Willan Publishing.

Loper, A. (2002). Adjustment to prison of women convicted of possession, trafficking, and non-drug offenses. Journal of Drug Issues, 32 (4), 10331050.

Mayer, R., \& Ouellet, F. (1991). Méthodologie de recherche pour les intervenants sociaux. Boucherville: Editions Gaëtan Morin.

Mullen, P. D., Cummins, A. G., Velaquez, M. M., Von Sternberg, K., \& Carvajal R. (2003). Jails as important but constrained venues for addressing women's health. Family $\mathfrak{F}$ Community Health, 26 (2), 157-168.

Mullings, J. L., Marquart, J. M., \& Diamond, P. M. (2001). Cumulative continuity and injection drug use among women: A test of the downward spiral framework. Deviant Behavior, 22 (3), 211-238.

Pate, K. (2002). Recension des dix dernières années relativement aux femmes condamnées à des peines fédérales: de mal en pis. Criminologie, 35 (2), 147158.

Phillips, J. A., Nixon, S. J., \& Pfefferbaum, B. (2002). A comparison of substance abuse among female offender subtypes. Journal of the American Academy of Psychiatry and the Law, 30 (4), 513-519.

Plourde, C. (2000). Consommation de substances psychoactives dans les pénitenciers québécois. Montréal: Thèse de doctorat, Université de Montréal.

Plourde, C., \& Brochu, S. (2002a). Drugs in Prison: A Break in the Pathway. Substance Use and Misuse, 37, 47-63

Plourde, C., \& Brochu, S. (2002b). Drogue et alcool durant l'incarcération: examen de la situation des pénitenciers québécois. Revue Canadienne de Criminologie, 44 (2), 209-240. 
Plourde, C., Brochu, S., Gendron, A., \& Couvrette, A. (en préparation). Substance Addiction and Incarceration: Situation of women incarcerated in Canadian Federal Institutions. Addictive Behaviors.

Seyle, H. (1953). The Stress of Life. New York: McGraw-Hill.

Stanley, E., \& Byrne, S. (2000). Mothers in prison: Coping with separation from children. In AIC/DCS Conference on Women in Corrections, Adelaide.

Staton, M., Leukefeld, C., \& Webster, J. M. (2003). Substance use, health, and mental health: Problems and service utilization among incarcerated women. International Journal of Offender Therapy and Comparative Criminology, 47 (2), 224-239.

Surratt (2003). Parenting Attitudes of Drug-Involved Women Inmates. The Prison Journal, 83 (2), 206-220.

Tonry, M., \& Petersilia, J. (1999). American Prisons at the beginning of the twenty-first century. In M. Tonry \& J. Petersilia (ed.), Crime and Justice, a review of research, 26, 1-16.

Vacheret, M., \& Lemire, G. (1998). L'univers de la prison à l'aube du $21^{e}$ siècle. Une analyse de pénitenciers canadiens. Montréal: Cahiers du Centre International de Criminologie Comparée de l'Université de Montréal.

Walters, G. D. (1996). The natural history of substance misuse in an incarcerated criminal population. Journal of Drug Issues, 26 (4), 943-959.

Withers, L. (2002). De l'autre coté de la barrière: les familles, les services correctionnels et la justice réparatrice. Ottawa: Regroupement canadien pour le bien-être des familles des prisonniers (ères).

Weekes, J. R., Vanderburg, S. A., \& Millson, W. (1995). Questionnaire informatisé sur le mode de vie (QIMV).Ottawa: Service correctionnel du Canada.

Zamble, E., \& Porporino, F. J. (1988). Coping, Behavior, and Adaptation in Prison Inmates. New York: Springer-Verlag. 